\title{
A CONVERSE OF THE HILBERT SYZYGY THEOREM
}

\author{
CHARLES CHING-AN CHENG AND JAY SHAPIRO
}

\begin{abstract}
The following converse of the Hilbert Syzygy Theorem is proved. Suppose $K$ is a noetherian commutative ring with identity that has finite global dimension, and suppose that $M$ is a finitely generated abelian cancellative monoid. If gl $\operatorname{dim} K M=n+\mathrm{gl} \operatorname{dim} K$ then $M$ is of the form $\left(\times_{i=1}^{n} M_{i}\right) \times H$ where $M_{i} \cong \mathbf{Z}$ or $\mathbf{N}$ and where $H$ is a finite group with no $K$-torsion.
\end{abstract}

Let $R$ be a ring with identity and let $M$ be a monoid. We shall denote by $R M$ the monoid ring of $M$ over $R$. The following theorems are well known (see, for example, $[2,3,8,10,11])$.

1 (Hilbert Syzygy). Suppose $M$ is either $\mathbf{N}$, the additive monoid of natural numbers, or $\mathbf{Z}$, the additive group of integers. Then

$$
\mathrm{gl} \operatorname{dim} R M=1+\mathrm{gl} \operatorname{dim} R \text {. }
$$

2 (MASCHKE). Suppose $M$ is a finite group. Then

$$
\begin{aligned}
\mathrm{gl} \operatorname{dim} R M & =\mathrm{gl} \operatorname{dim} R, & & \text { if }|M| \text { is invertible in } R, \\
& =\infty, & & \text { otherwise. }
\end{aligned}
$$

Using the fact that $R\left(M_{1} \times M_{2}\right) \cong\left(R M_{1}\right) M_{2}$, one could combine the above results to yield the following.

(Generalized Syzygy). Suppose $R$ is a ring with identity and suppose $M$ is a monoid of form

$$
\left(\sum_{i=1}^{n} M_{i}\right) \times H,
$$

where $M_{1} \cong \mathbf{N}$ or $\mathbf{Z}$ and where $H$ is a finite group with no $R$-torsion (i.e. $|H|$ is invertible in $R$ ). Then

$$
\mathrm{gl} \operatorname{dim} R M=n+\mathrm{gl} \operatorname{dim} R \text {. }
$$

In this paper we shall prove the following converse of this result.

THEOREM A. Suppose $K$ is a noetherian commutative ring with identity such that $\mathrm{gl} \operatorname{dim} K<\infty$, and suppose that $M$ is a finitely generated abelian cancellative monoid.

Received by the editors August 11, 1982 and, in revised form, December 13, 1982.

1980 Mathematics Subject Classification. Primary 13D05, 16A60, 18G20; Secondary 20J99, 20 M25.

Key words and phrases. Monoid, cohomological dimension, global dimension, Hochschild dimension. 
If $\mathrm{gl} \operatorname{dim} K M=n+\mathrm{gl} \operatorname{dim} K$ then $K$ is of form

$$
\left(\chi_{i=1}^{n} M_{i}\right) \times H
$$

where $M_{i} \cong \mathbf{N}$ or $\mathbf{Z}$ and where $H$ is a finite group with no $K$-torsion.

Remark. Combining the Generalized Syzygy Theorem with Theorem A one has the following: Suppose $K$ is a noetherian commutative ring with identity and suppose $M$ is a finitely generated abelian cancellative monoid. Then $g l \operatorname{dim} K M<\infty$ if and only if gl $\operatorname{dim} K<\infty$ and $M$ is of form (1).

Using Theorem A, we obtain a corresponding result for the $K$-Hochschild dimension $\operatorname{dim}_{K} M$ of $M$ for any commutative ring $K$ (not necessarily noetherian).

THEOREM B. Suppose $K$ is a commutative ring with identity and suppose that $M$ is a finitely generated abelian cancellative monoid. Then $\operatorname{dim}_{K} M=n<\infty$ if and only if $M$ is of form (1).

1. Proof of Theorem A. Throughout $M$ will denote a monoid and $\hat{M}$ will denote its group reflection [4]. Suppose $M$ is abelian and cancellative. Then $\hat{M}$ is simply the group of quotients of $M$, i.e. $\hat{M}$ has elements of form $a / b, a, b \in M$, with the obvious multiplication. In this case, the canonical map $M \rightarrow \hat{M}$, taking $a \in M$ to $a / 1$, is injective and so one may assume that $M \subseteq \hat{M}$. Note that the group ring $R \hat{M}$ over any ring $R$ is simply the localization of $R M$ at the central multiplicative set $M$. Therefore

$$
\text { gl } \operatorname{dim} R \hat{M} \leqslant \mathrm{gl} \operatorname{dim} R M .
$$

LEMMA 1. If Theorem A is true for any noetherian domain $K$ then it is true for any noetherian commutative ring $K$.

Proof. Suppose gl $\operatorname{dim} K M=n+$ gl $\operatorname{dim} K$ for a noetherian commutative ring $K$. Using [9, Theorems 164, 168] together with the fact that gl $\operatorname{dim} K<\infty$, one sees that $K$ is a direct product of finitely many noetherian domains $D_{j}$. This implies that $K M$ is a direct product of the monoid rings $D_{j} M$. Hence

$$
\text { gldim } D_{j} M \leqslant \operatorname{gldim} K M \text { and } \operatorname{gldim} D_{j} \leqslant \operatorname{gldim} K \text {. }
$$

Therefore, as $\mathrm{gl} \operatorname{dim} D_{j} \leqslant \mathrm{gl} \operatorname{dim} D_{j} M$, one has $\mathrm{gl} \operatorname{dim} D_{j} M=m+\mathrm{gldim} D_{j}$. Thus $M$ must be of form (1) where $M_{i} \cong \mathbf{N}$ or $\mathbf{Z}$ using the Generalized Syzygy Theorem and where $H$ is a finite group with no $D_{j}$-torsion (and, therefore, no $K$-torsion).

Proof of Theorem A. By Lemma 1, we may assume that $K$ is a noetherian domain. Since $\mathrm{gl} \operatorname{dim} K<\infty$ and $g l \operatorname{dim} K M=n+\operatorname{gl} \operatorname{dim} K$, one has $g l \operatorname{dim} K \hat{M}<$ $\infty$ by (2). Since $M$ is finitely generated as a monoid, $\hat{M}$ is finitely generated as a group and so $\hat{M} \cong \mathbf{Z}^{t} \times H$ where $H$ is a finite group with no $K$-torsion by the Generalized Syzygy Theorem. Let $G$ be the group of invertible elements of $M$. Then $G$ is a subgroup of $\hat{M}$ and, therefore, $G=G_{F} \times G_{T}$ where $G_{F}$ is a maximal torsion-free subgroup and $G_{T}$ the torsion subgroup of $G$.

Let $N$ be the set of all noninvertible elements of $M$. We will call an element of $N$ irreducible if whenever it factors in $M$ then one of those factors is a unit in $M$. Define 
an equivalence relation on the irreducible elements as follows: $a \sim b$ if and only if there exists $g \in G$ with $a g=b$. Choose a set $S$ of irreducible elements of $M$, one from each equivalence class of $\sim$. Since $K$ is noetherian and $M$ finitely generated abelian, $K M$ is noetherian. Thus every element of $M$ factors into a product of irreducible elements, in particular one has $M=\langle G, S\rangle$. It then follows that any set generating $M$ contains at least one irreducible from each equivalence class of $\sim$. Since $M$ is finitely generated, $S$ must be finite.

Let $S=\left\{x_{1}, \ldots, x_{s}\right\}$, and let $r=\operatorname{rank} G$. It is not hard to show that the rank of the group reflection of $\left\langle G_{F}, S\right\rangle$ is equal to that of $\hat{M}$. Thus $s+r \geqslant t$. We will show that equality holds. Let $p=K N+I \subseteq K M$ where $K N$ denotes the set of all linear combinations of elements of $N$ over $K$ and where $I$ denotes the ideal of $K M$ generated by elements of the form $1-g, g \in G_{T}$. Since $K M / p \cong K G_{F}$ is a domain, $p$ is a prime ideal of $K M$. The following result concerning Krull dimension is needed.

Lemma 2 (GILMER [7], ARNOLD-GILMER [1]). If $K$ is a commutative ring with identity and if $M$ is an abelian cancellative monoid, then

$$
\text { Krull } \operatorname{dim} K M=\text { Krull } \operatorname{dim} K \hat{M}=\operatorname{rank} \hat{M}+\text { Krull } \operatorname{dim} M .
$$

Hence

$$
\begin{aligned}
\text { Krull } \operatorname{dim} K M_{p} & \leqslant \text { Krull } \operatorname{dim} K M-\mathrm{Krull} \operatorname{dim} K M / p \\
& =\text { Krull } \operatorname{dim} K \hat{M}-\text { Krull } \operatorname{dim} K G_{F} \\
& =t-r .
\end{aligned}
$$

Therefore, in order to establish that $t=r+s$, it is enough to show that Krull $\operatorname{dim} K M_{p} \geqslant s$. Since $K M_{p}$ is regular, one only needs to show that $p_{p}$ requires $s$ generators by the theorem of Auslander-Buchsbaum-Serre. Let $D$ be the set of all decomposable elements of $M$. Then $K M / K D=K G\left[x_{1}, \ldots, x_{s}\right] /\left(x_{i} x_{j}\right)$. Note that we have an obvious map $K G \rightarrow K G_{F} \rightarrow Q$ where $Q$ is the field of quotients of $K G_{F}$ and that this induces a map from $K M / K D \rightarrow B$ where $B=Q\left[x_{1}, \ldots, x_{s}\right] /\left(x_{i} x_{j}\right)$. Composing the latter with the canonical projection we obtain a map $\phi: K M \rightarrow B$. Let $q$ be the ideal of $B$ generated by $x_{1}, \ldots, x_{s}$. Then the inverse image of $q$ is $p$ and so there is an induced map $\phi^{\prime}: K M_{p} \rightarrow B_{q}$. Since $\phi^{\prime}\left(p_{p}\right)$ generates $q_{q}$ we only need to show that $q_{q}$ needs $s$ generators. But this is not hard to show since $q$ requires $s$ generators and that $Q$ is a field.

Hence $t=s+r$. Consider the map $f: \mathbf{N}^{s} \times G \rightarrow M$ induced by the elements $x_{i} \in S$ and the inclusion $G \subseteq M$. It is onto and induces $g: Z^{s} \times G \rightarrow \hat{M}$ whose kernel is torsion since $\operatorname{rank}\left(\mathbf{Z}^{s} \times G\right)=\operatorname{rank} \hat{M}$. But the restriction of $g$ to $G_{T}$ is the inclusion $G_{T} \subseteq \hat{M}$. Therefore, $g$ is injective and so is $f$. Hence $M$ is of form (1).

2. The Hochschild dimension of an abelian monoid. Let $K$ be a nonzero commutative ring with identity and let $M$ be a monoid. The $K$-Hochschild dimension of $M$ is defined to be the projective dimenson of $K M$ over its enveloping algebra and we shall denote it by $\operatorname{dim}_{K} M$. Clearly $\operatorname{dim}_{K} M=\operatorname{dim}_{K} M^{\text {op }}$ and it is easy to show that $\operatorname{dim}_{K} M \leqslant \operatorname{dim}_{L} M$ whenever there is a ring homomorphism $L \rightarrow K$.

Recall that if $R$ is a ring with identity then the $R$-cohomological dimension of $M$ is the projective dimension of the trivial $R M$-module $R$ where the $R M$-action on $R$ is 
defined by $x r=r$ for $x \in M, r \in R$. In general, $c d_{K} M \leqslant \operatorname{dim}_{K} M$ (see [3, p. 185]). When $M$ is a group, the equality holds (see [3, p. 195]) and, thus, if $M$ has $K$-torsion, then $\operatorname{dim}_{K} M=\infty$.

LEMMA 3 (EILENBERG-ROSENBERG-ZELINSKY [6]).

$$
\text { l.gl } \operatorname{dim} K M \leqslant \operatorname{dim}_{K} M+\text { l.gl } \operatorname{dim} K \text {. }
$$

REMARK. Readers should consult $[10$, p. 62] for a generalization of the above result as well as proof without the use of spectral sequences.

The following lemma is similar to the one due to Swan concerning $c d_{K} M$. The proof is exactly the same.

Lemma 4 (SWAN [12]). For a commutative ring $K$ and a monoid $M$ there exists a prime field $F$ such that $\operatorname{dim}_{F} M \leqslant \operatorname{dim}_{K} M$.

Lemma 5. Suppose $M_{1}$ and $M_{2}$ are monoids. Then $\operatorname{dim}_{K} M_{1} \times M_{2} \leqslant \operatorname{dim}_{K} M_{1}+$ $\operatorname{dim}_{K} M_{2}$.

Proof. This follows from [6, Remark 3, p. 75] together with the observation that

$$
K M_{1} \otimes_{K} K M_{2} \cong K\left(M_{1} \times M_{2}\right) .
$$

LEMma 6. (a) If $M \cong \mathbf{N}$ or $\mathbf{Z}$ then $\operatorname{dim}_{K} M=1$.

(b) If $M$ is a finite group with no K-torsion, then $\operatorname{dim}_{K} M=0$.

Proof. (a) is well known (see, e.g., [10, p. 119]) and (b) follows from the second paragraph of this section.

Proof of Theorem B. Suppose $M$ is of form (1). Then, using Lemmas 5 and 6 , one has

$$
\operatorname{dim}_{K} M \leqslant \sum_{i} \operatorname{dim}_{K} M_{i}+\operatorname{dim}_{K} H=n .
$$

By Lemma $3, n+\operatorname{gldim} K \leqslant \operatorname{dim}_{K} M+\operatorname{gl} \operatorname{dim} K$ for any commutative ring $K$. Thus $n \leqslant \operatorname{dim}_{K} M$. Hence $\operatorname{dim}_{K} M=n$. Conversely, if $\operatorname{dim}_{K} M=n$ then $\operatorname{dim}_{F} M \leqslant n$ for some prime field $F$ and so $g l \operatorname{dim} F M \leqslant \operatorname{dim}_{F} M \leqslant n$. Hence, by Theorem A, $M$ is of the form $\left(\times_{i=1}^{m} M_{i}\right) \times H$ where $H$ is a finite group with no $F$-torsion. Using the "if" part of Theorem B just proved, one sees that $m=n$ and $H$ has no $K$-torsion.

In the following, we shall exhibit examples to show that some condition on the abelian monoid $M$ is necessary in Theorems $\mathrm{A}$ and $\mathrm{B}$.

ExAmple 1. Let $M$ be the additive group $\mathbf{Q}$ of rationals. Then $M$ is infinitely generated (as an abelian monoid) and, thus, is not of form (1). However, $\operatorname{dim}_{K} \mathbf{Q}=2$ and $\mathrm{gl} \operatorname{dim} K \mathbf{Q}=2+\mathrm{gl} \operatorname{dim} K$ for any noetherian commutative $\operatorname{ring} K$ (see, e.g., [2]).

EXAmple 2. It can be shown that if $M$ is a finite abelian monoid such that every element is an idempotent, then $\operatorname{dim}_{K} M=0$. In this case $\mathrm{gl} \operatorname{dim} K M=\mathrm{gl} \operatorname{dim} K$ but $M$ does not have to be cancellative and, thus, need not be of form (1).

AcKnowledgement. The authors would like to thank Professor R. G. Swan for simplifying a portion of the proof of Theorem A. 


\section{REFERENCES}

1. J. T. Arnold and R. Gilmer, The dimension theory of commutative semigroup rings, Houston J. Math. 2 (1976), 299-313.

2. S. Balcerzyk, The global dimension of the group rings of abelian groups. III. Fund. Math. 67 (1970), $241-250$.

3. H. Cartan and S. Eilenberg, Homological algebra, Princeton Univ. Press, Princeton, N. J., 1956.

4. C. C. Cheng and J. Shapiro, Cohomological dimension of an abelian monoid. Proc. Amer. Math. Soc. 80 (1980), 547-551.

5. C. C. Cheng and R. Wong, Hereditary monoid rings, Amer. J. Math. 104 (1982), 935-942.

6. S. Eilenberg, A. Rosenberg and D. Zelinsky. On the dimension of modules and algebras. VIII. dimension of tensor products, Nagaya Math. J. 12 (1957), 71-93.

7. R. Gilmer, A two-dimensional non-Noetherian factorial ring. Proc. Amer. Math. Soc. 44 (1974). $25-30$.

8. I. Kaplansky, Fields and rings, Univ. of Chicago Press, Chicago, Ill., 1969.

9. Commutative rings, Allyn and Bacon, Boston, Mass., 1970.

10. B. Mitchell, Rings with several objects, Adv. in Math. 8 (1972), 1-161.

11. J. Rotman, An introduction to homological algebra, Academic Press, New York. 1979.

12. R. G. Swan, Groups of cohomological dimension one. J. Algebra 12 (1969), 585-610.

Department of Mathematics, The Institute for Advanced Study. Princeton. New Jersey 08540

Department of Mathematics, Oakland University, Rochester, Michigan 48063 (Current address of C. C. Cheng)

Department of Mathematics, George Mason University, Fairfax, Virginia 22030 (Current address of Jay Shapiro) 